\title{
Thromboelastographic profile of goat blood after the experimental injury of the femoral artery and use of QuikClot gauze and Celox gauze dressings
}

\author{
P. Sobiech ${ }^{1}$, Z. Adamiak ${ }^{2}$, P. Holak ${ }^{2}$, P. Jastrzębski ${ }^{3}$, J. Rogowski ${ }^{4}$, \\ M. Brzeziński ${ }^{4}$, K. Bury ${ }^{4}$, M. Jałyński ${ }^{2}$, W. Baumgartner ${ }^{5}$ \\ ${ }^{1}$ Department of Internal Diseases, Faculty of Veterinary Medicine, \\ University of Warmia and Mazury in Olsztyn, Oczapowskiego 14, 10-957 Olsztyn, Poland \\ ${ }^{2}$ Department of Surgery and Radiology, Faculty of Veterinary Medicine, \\ University of Warmia and Mazury in Olsztyn, Oczapowskiego 14, 10-957 Olsztyn, Poland \\ ${ }^{3}$ Police Academy, Institute of Basic Security, Piłsudskiego 25, 12-100 Szczytno, Poland \\ ${ }^{4}$ Department and Clinic of Cardiac and Vascular Surgery, Faculty of Medicine, \\ Medical University of Gdańsk, Debinki 7, 80-211 Gdańsk, Poland \\ ${ }^{5}$ Vienna University of Veterinary Medicine, Clinic for Ruminants, Veterinaerplatz 1, 1210 Vienna, Austria
}

\begin{abstract}
The aim of this study was to evaluate the suitability of thromboelastometry for the analysis of blood test results in goats after the use of hemostatic dressings to control massive bleeding. The study was carried out on 12 goats, 6 animals in each of two subgroups. In all experimental animals incision of the femoral artery was performed, and bleeding was controlled with QuikClot gauze in the first group and Celox gauze in the second group. Dressings were applied for 60 minutes. Blood samples for thromboelastometry were collected from the jugular vein before the incision and $60 \mathrm{~min}$ after the application of a dressing. Clotting time (CT), clot formation time (CFT), maximum clot firmness (MCF) and $\alpha$ angle $\left({ }^{\circ}\right)$ were measured in three standard ROTEM assays (system with generation of reaction curve, numerical parameters and size of the blood clot): intrinsic coagulation pathway (INTEM), extrinsic coagulation pathway (EXTEM) and functional fibrinogen (FIBTEM). Complete hemostasis of the injured femoral artery was found in all goats. No significant differences between pre- and post-incision thromboelastometric parameters were found in any tests in any of the groups, which indicates that the use of dressings was not associated with blood coagulation disorders. This study is the first to describe the use of thromboelastometry in goats for the assessment of clot formation and hemostatic disorders.
\end{abstract}

Key words: hemostatic dressing, goats, thromboelastometry

Correspondence to: P. Sobiech, e-mail: psobiech@uwm.edu.pl 


\section{Introduction}

The analysis of the coagulation system and diagnosis of its disorders are major elements determining hemostasis. Presently, there is no gold standard for the diagnosis of hypercoagulability. Until recently, both human medicine and veterinary medicine used the routine determination of coagulation parameters, such as thrombin time, prothrombin time, activated partial thromboplastin time, antithrombin III activity or D-dimer concentration (Gentry 2004). Changes in these parameters were reported in cows during calving (Hackner et Schaer 2010), in cows with indigestion (Gokce et al. 2007, Rhee et al. 2008) and in newborn calves with respiratory and gastrointestinal diseases (Heuwieser et al. 1990, Gokce et al. 2006). The most common form of hemostasis dysfunction connected with these disorders observed in large animals is a syndrome known variously as DIC (disseminated intravascular coagulation), consumption coagulopathy, defibrination syndrome or intravascular coagulation fibrinolysis. DIC is caused by excessive activation of coagulation, either regionally or throughout the entire body. DIC and hypercoagulable states are acquired disorders of hemostasis in animals that occur as a consequence of severe disease that induces systemic inflammation (Hackner and Schaer 2010). Determination of coagulation parameters should be introduced into routine veterinary practice; such parameters are very reliable prognostic indicators which are helpful in diagnosis of the degree of health disorders and in making decisions about further treatment or surgical intervention.

Thromboelastometry is one of the recently developed diagnostic methods, and allows for the rapid analysis of hemostasis and changes in the coagulation and fibrinolysis of whole blood. In contrast to traditional parameters of hemostasis, thromboelastometry also takes into account the function of both cellular and soluble components of coagulation and how they interact with each other in generating clots (Levrat et al. 2008). Because of this, thromboelastometry has gained importance in human medicine and surgery (Cammerer et al. 2003, Johnson et al. 2012). The results of thromboelastometric tests are presented in numerical and graphical formats, and interpreted using comparative data analysis. Reports on the use of thromboelastometry and thromboelastography in diagnosing coagulation disorders in animals are very limited and mainly concern experimental studies (Sobiech et al. 2008, Nowshad et al. 2012, Goggs et al. 2014). The ROTEM ${ }^{\circledR}$ system, which generates a reaction curve, numerical parameters and size of the (formed) blood clot, is one of the most popular tools used in thromboelastometric and thromboelastographic tests (Irmak et al. 2006). The addition of special re- agents to a blood sample initiates the intrinsic (INTEM assay) or extrinsic (EXTEM assay) pathway of blood coagulation. This allows for an overall analysis of the coagulation system performance. The INTEM assay, where the intrinsic pathway is activated by ellagic acid, is used for the analysis of clotting factors (XII, XI, IX, VIII, X, V, II, and I), platelets and fibrinolysis. The EXTEM assay, where the extrinsic pathway is activated by thromboplastin (tissue factor), is used for the analysis of clotting factors (VII, X, V, II and I), platelets and fibrinolysis. In the FIBTEM assay platelet contribution to clot formation can be inhibited by the addition of another reagent, i.e. cytochalasin $\mathrm{D}$, and therefore fibrinogen deficiency or fibrin polymerization disorders can be detected. One of the main parameters determined by thromboelastometry is coagulation time (CT), expressed in seconds. $\mathrm{CT}$ is the latency time from adding the reagent to blood until a $2 \mathrm{~mm}$ clot starts to form, and represents the stage from the start of the coagulation to the formation of the first fibrin fibers and activated platelets. The second important parameter, clot formation time (CFT), is expressed in seconds and denotes the time required for the formation of a stable clot with the contribution of platelets and fibrin. At this time interval the clot increases from $2 \mathrm{~mm}$ to $20 \mathrm{~mm}$. The alpha angle is the slope of the tangent to the reaction curve starting at the CT endpoint $(2 \mathrm{~mm})$. Maximum clot firmness (MCF), expressed in millimeters, is the greatest vertical amplitude of the trace. It reflects the strength of the fibrin and platelet clot.

Hemostatic dressings have been developed for use in cases of massive and difficult to control bleeding resulting in rapid death. Commercially available hemostatic dressings are able to stop bleeding from a large artery or vein within minutes of their application onto the wound. Hemostatic dressings such as QuikClot gauze and Celox gauze are widely used in human emergency medicine, especially on the battlefield (Gegel et al. 2013). In veterinary medicine, hemostatic dressings have been mainly used in experimental studies on the swine model (Jackson et al. 2009, Adamiak et al. 2014).

To date, no reports on thromboelastometric studies in goats have been published. The aim of our study was to evaluate the suitability of thromboelastometry for analysis of the coagulation system in goats in which massive bleeding from the femoral artery was controlled with selected hemostatic dressings.

\section{Materials and Methods}

Animals used in this study were in accordance with Polish Council of Animal care standards and ap- 
Table 1. Thromboelastometer results in goats using QuikClot gauze dressing $(n=6, \bar{x}+\mathrm{SD})$.

\begin{tabular}{lcccc}
\hline Test & CT $(\mathrm{s})$ & CFT $(\mathrm{s})$ & MCF $(\mathrm{mm})$ & Alpha $\left({ }^{\circ}\right)$ \\
\hline INTEM b.o. & $181 \pm 45.3$ & $33,5 \pm 12.8$ & $83 \pm 7.8$ & $83.5 \pm 3.2$ \\
INTEM p.o. & $203.5 \pm 58.2$ & $35 \pm 14.1$ & $81 \pm 8.2$ & $83 \pm 5.3$ \\
EXTEM b.o. & $61,5 \pm 15.8$ & $34,5 \pm 11.3$ & $79 \pm 5.8$ & $85 \pm 4.9$ \\
EXTEM p.o. & $58 \pm 17.4$ & $35,5 \pm 12.3$ & $85 \pm 6.3$ & $86 \pm 2.7$ \\
FIBTEM b.o. & $38.5 \pm 20.2$ & $28 \pm 9.2$ & $67 \pm 4.9$ & $85 \pm 2.5$ \\
FIBTEM p.o. & $42.5 \pm 14.1$ & $31.5 \pm 13.1$ & $66.5 \pm 6.6$ & $86 \pm 3.4$ \\
\hline
\end{tabular}

b.o. - before operation, p.o. - post operation

Table 2. Thromboelastometer results in goats with using Celox gauze dressing $(n=6, \bar{x}+\mathrm{SD})$.

\begin{tabular}{rcccc}
\hline Test & CT $(\mathrm{s})$ & CFT $(\mathrm{s})$ & MCF $(\mathrm{mm})$ & Alpha $\left({ }^{\circ}\right)$ \\
\hline INTEM b.o. & $169.2 \pm 34.8$ & $45.5 \pm 13.2$ & $82.3 \pm 6.7$ & $81.4 \pm 4.4$ \\
INTEM p.o. & $181.5 \pm 45.3$ & $40.2 \pm 11.9$ & $82.3 \pm 8.8$ & $82.1 \pm 5.2$ \\
EXTEM b.o. & $62.5 \pm 13.9$ & $44.5 \pm 12.8$ & $82.2 \pm 7.1$ & $83.3 \pm 4.9$ \\
EXTEM p.o. & $66.4 \pm 16.1$ & $41.3 \pm 15.4$ & $81.2 \pm 7.7$ & $79.5 \pm 3.3$ \\
FIBTEM b.o. & $57.2 \pm 12.9$ & $37.5 \pm 14.6$ & $61.5 \pm 5.3$ & $84.5 \pm 3.7$ \\
FIBTEM p.o. & $60.2 \pm 17.2$ & $37.1 \pm 11.7$ & $59.3 \pm 5.8$ & $83.3 \pm 4.1$ \\
\hline
\end{tabular}

b.o. - before operation, p.o. - post operation

proved under the Local Ethics Commission of the University of Warmia and Mazury in Olsztyn (02/2015). All goats were handled humanely in compliance with the Policy on the Humane Care and Use of Laboratory Animals. Twelve male Alpine goats, age 6-7 years and $34-35 \mathrm{~kg}$ body weight (BW), were used in the study. The animals were sedated with 0.1 mg xylazine/kg BW i.m. (VetaXyl, Vet Agro, Poland), and $0.1 \mathrm{mg}$ butorphanol $/ \mathrm{kg} \mathrm{BW}$ i.m. (Butomidor, Richter Pharma, Austria). General anesthesia was induced with $6 \mathrm{mg}$ ketamine $/ \mathrm{kg}$ BW i.v. (Bioketan, Vetoquinol, Poland) and $0.2 \mathrm{mg}$ diazepam $/ \mathrm{kg} \mathrm{BW}$ (Relanium $5 \mathrm{mg} / \mathrm{ml} \mathrm{WZF} \mathrm{Polfa} \mathrm{S.A.,} \mathrm{Poland),} \mathrm{and}$ further maintained with $1 \mathrm{mg}$ propofol $/ \mathrm{kg} / \mathrm{min}$ (Scanofol, Scan Vet, Poland).

The animals were divided randomly into 2 groups of equal numbers. Blood samples for thromboelastometric tests were collected from all animals from the jugular vein before the surgical procedure and 60 min after hemostatic dressing application. 50 $\mathrm{mm}$-long transverse incisions were then made through the skin across the entire femoral artery. After blunt dissection of the muscles the left femoral artery was injured at groin level. In the first group of six animals bleeding was controlled using QuikClot rolled gauze, $7.62 \mathrm{~cm}$ wide and $365 \mathrm{~cm}$ long, impregnated with hemostatic agent (kaolin). The hemostatic dressing was applied directly onto the injured vessel, immediately after the incision of the femoral artery, tightly filling the space between dissected muscles, and left inside the wound for 60 minutes. The same surgical procedure was followed in the second group, but Celox rolled gauze, $7.62 \mathrm{~cm}$ wide and $305 \mathrm{~cm}$ long, impregnated with chitosan, was used for bleeding control.

Ringer's lactate solution $(1.000 \mathrm{ml})$ was administered during surgery for $60 \mathrm{~min}$ in each animal. The animals were euthanized directly after blood sampling using $140 \mathrm{mg}$ euthasol/kg BW i.v. (Virbac).

Blood samples for tromboelastography examination for the ROTEM system (Pentapharm, D) were collected in $3 \mathrm{ml}$ tubes containing buffered sodium citrate. Three standard ROTEM assays were performed: intrinsic coagulation pathway - INTEM, extrinsic coagulation pathway - EXTEM, and functional fibrinogen - FIBTEM. The following parameters were evaluated for each coagulation profile: clotting time (CT), clot formation time (CFT), maximum clot firmness (MCF) and $\alpha$ angle $\left({ }^{\circ}\right)$.

The values of thromboelastometric parameters were presented as means $(\bar{x})$ and standard deviation (SD). The significance of differences between test results before and after surgical procedure was determined using the Newman-Keulus test at a confidence level of $p \leq 0.01$.

\section{Results}

In all animals hemostatic dressings were removed in minute 60 after the surgical procedure. Complete hemostasis of the injured femoral artery, demonstrating the efficiency of both dressings, was found in all goats.

The INTEM tests in goats treated with QuikClot gauze indicated a slightly longer clotting time $(\mathrm{CT})$ after the procedure, but no significant changes in 
other parameters (CFT, MCF and alpha angle) were found (Table 1). The EXTEM test demonstrated only an insignificant decrease in MCF after the procedure, and there were no inter-assay differences in the FIBTEM test (Table 1). The INTEM test in goats treated with the Celox gauze dressings revealed an insignificant increase in clotting time after the procedure, but values for other parameters were similar before and after the procedure (Table 2). There were no significant differences between all the analyzed parameters before and after the procedure in the EXTEM and FIBTEM tests (Table 2).

\section{Discussion}

All thromboelastometric tests (INTEM, EXTEM, FIBTEM) carried out in goats before the surgical procedure demonstrated shorter CT than that reported for cattle (Falco et al. 2011). No significant differences were found between coagulation times measured before and after the procedure, regardless of the type of dressing used (QickClot gauze or Celox gauze). CT is a useful indicator of the activity of the coagulation factors involved in the formation of thrombin in the extrinsic or intrinsic pathway (depending on the activator) and activator-inhibitor equilibrium. Prolonged CT can indicate a deficiency of clotting factors or the presence of antithrombotic drugs in the blood. The lack of significant differences between pre- and post-procedure blood samples suggests that both dressings had no negative effect on the efficiency of the coagulation system in the experimental animals. It is worth emphasizing, that there are no reference values for goats available up to now in the literature worldwide concerning thromboelastometry parameters for goats.

In all tests, samples of blood collected from animals before and after the procedure did not differ for clot formation time. Comparative analysis demonstrated that CFT in goats is clearly shorter than in cattle (Falco et al. 2011). Major factors affecting CFT are the contribution of platelets in clot formation, fibrinogen level and fibrin polymerization. Studies performed in calves (Borrelli et al. 2013) revealed reduced values of CFT in all tests in animals with hypercoagulability associated with the administration of steroid drugs. No such disorders were identified in our study.

The alpha angle expresses the velocity of clot formation, and depends, similarly to CFT, on the contribution of platelets in clot formation, the concentration of fibrinogen and fibrin polymerization. Low values of alpha angle $\left(^{\circ}\right)$ indicate hypocoagulation of blood and high values indicate hypercoagulation. Values of the alpha angle found in our study were slightly higher than those reported for cattle and calves (Falco et al. 2011, Borrelli et al. 2013), but there were no inter-assay differences, which suggests a lack of disorders associated with the treatments carried out.

There were no significant differences between MCF in all goats, but the values were clearly higher than those reported for cattle (Falco et al. 2011). MCF is determined by platelet quality and count, fibrinogen level, fibrin polymerization, factor XIII and fibrinolysis. In humans, low MCF values (Jastrzębski et al. 2014) suggest the need to introduce treatment with platelet concentrates or fibrinogen. In patients with reduced MCF values, differential diagnosis employing both EXTEM and FIBTEM tests allows for discrimination between platelet disorders affecting clot firmness, and abnormal fibrinogen levels or fibrin polymerization (Levrat et al. 2008).

The effectiveness and usefulness of QuikClot gauze and Celox gauze in the rapid control of massive bleeding has been repeatedly confirmed in experimental studies (Paltrinieri et al. 2008), in hospital settings and on the battlefield in direct warfare (Devlin et al. 2011, Rall et al. 2013). Our study demonstrated the efficacy of both dressings in the control of bleeding from the injured femoral artery. To date, no reports have been available on the use of hemostatic QuikClot gauze and Celox gauze in goats. Previous experimental studies were carried out in swine (Adamiak et al. 2014) and ovine models (Gokce et al. 2006). Our experiment was designed to investigate the efficiency of hemostatic dressings applied for $60 \mathrm{~min}$ to control massive bleeding. In most studies hemostatic dressings were kept inside the wound for $30 \mathrm{~min}$ (Johansson et al. 2012, Gegel et al. 2013). Unlike other researchers (Luddington 2005), we applied dressings immediately after the incision of the femoral artery, assuming that the lack of drop in blood pressure may negatively affect the efficiency of hemostatic dressings. Some reports (Gegel et al. 2013) indicate the efficiency of QuikClot gauze and Celox gauze in controlling massive bleeding within a $30 \mathrm{~min}$ application. The present study demonstrated that both hemostatic dressings applied for $60 \mathrm{~min}$ immediately after the injury, i.e. when no drop in blood pressure occurred, are equally effective, as reported by other researchers (Paltrinieri et al. 2008).

\section{Conclusion}

The study demonstrated that in both experimental groups the hemostasis achieved using the QuikClot gauze and Celox gauze dressings had no negative effect on the blood coagulation system. No disorders of 
coagulation or fibrinolysis were observed. All analyzed parameters suggested that the blood clot formed in the region of the injured artery was stable, and thus strongly minimized the risk of rebleeding. Results show that thromboelastography is a valuable diagnostic method evaluating clot formation, its quality and lysis after the application of QuikClot gauze and Celox gauze in goats. Moreover, the thromboelastographic profile of blood and the lack of coagulation disorders after the use of hemostatic dressings indicate the very high degree of safety of both types of gauze.

\section{References}

Adamiak Z, Jastrzębski P, Pomianowski A, Otrocka-Domagała I, Holak P, Zhalniarovich Y, Przyborowska P, Głodek J (2014) Effect of 24 hour application of three hemostatic dressings to porcine thigh muscles. Pol J Vet Sci 17: 519-521.

Borrelli A, Bellino C, Bozzetta E, Bruno B, Falco S, Maurella C, Gianella P, Pezzolato M, Cagnasso A, D'Angelo A (2013) Thromboelastometry in veal calves to detect hemostatic variations caused by low doses of dexamethasone treatment. BMC Vet Res 9: 55.

Cammerer U, Dietrich W, Rampf T, Braun SL, Richter JA (2003) The predictive value of modified computerized thromboelastography and platelet function analysis for postoperative blood loss in routine cardiac surgery. Anesth Analg 96: 51-57.

Devlin JJ, Kircher S, Kozen BG, Littlejohn LF, Johnson AS (2011) Comparison of ChitoFlex, Celox, and Quik-Clot in control of hemorrhage. J Emerg Med 41: 237-245.

Falco S, Bruno B, Borrelli A, Cagnasso A, D'Angelo A (2011) Validation of thromboelastometry in cattle. In: Pugliese A, Gaiti A (eds) Veterinary Science. Springer-Verlag, Berlin, pp 91-96.

Gegel BT, Austin PN, Johnson AD (2013) An evidence-based review of the use of a combat gauze (QuikClot) for hemorrhage control. AANA J 81: 453-458.

Gentry PA (2004) Comparative aspects of blood coagulation. Vet J 168: 238-251.

Goggs R, Brainard B, de Laforcade AM, Flatland B, Hanel R, McMichael M, Wiinberg B (2014) Partnership on Rotational ViscoElastic Test Standardization (PROVETS): evidence-based guidelines on rotational viscoelastic assays in veterinary medicine. $\mathrm{J}$ Vet Emerg Crit Care 24: 1-22.

Gokce G, Gokce HI, Erdogan HM, Gunes V, Citil M (2006) Investigation of the coagulation profile in calves with neonatal diarrhoea. Turk J Vet Anim Sci 30: 223-227.
Gokce HI, Gokce G, Cihan M (2007) Alterations in coagulation profiles and biochemical and haematological parameters in cattle with traumatic reticuloperitonitis. Vet Res Commun 31: 529-537.

Hackner SG, Schaer BD (2010) Thrombotic disorders. In: Weiss DJ, Wardrop KJ (eds) Veterinary hematology. Wiley-Blackwell, pp 668-678.

Heuwieser W, Kautni J, Biesel M, Grunert E (1990) Coagulation profile of dairy cattle in the periparturient period. Zentralbl Veterinarmed A 37: 8-15.

Irmak K, Sen I, Col R, Birdane FM, Guzelbektes H, Civelek T, Yilmaz A, Turgut K (2006) The evaluation of coagulation profiles in calves with suspected septic shock. Vet Res Commun 30: 497-503.

Jackson GN, Ashpole KJ, Yentis SM (2009) The TEG vs the ROTEM thromboelastography/thromboelastometry systems. Anaesthesia 64: 212-215.

Jastrzębski P, Adamiak Z, Pomianowski A, Krystkiewicz W, Holak P, Sawicki S, Przyborowska P, Zhalniarovich Y, Gudzbeler G (2014) Response of the coagulation system after the application of hemostatic dressings in an animal model. Pol J Vet Sci 17: 725-727.

Johansson PI, Oliveri RS, Ostrowski SR (2012) Hemostatic resuscitation with plasma and platelets in trauma. J Emerg Trauma Shock 5: 120-125.

Johnson D, Westbrook DM, Phelps D, Blanco J, Bentley M, Burgert J, Gegel B (2014) The effects of QuikClot Combat Gauze on hemorrhage control when used in a porcine model of lethal femoral injury. Am J Disaster Med 9: 309-315.

Levrat A, Gros A, Rugeri L, Inaba K, Floccard B, Negrier C, David JS (2008) Evaluation of rotation thrombelastography for the diagnosis of hyperfibrinolysis in trauma patients. Br J Anaesth 100: 792-797.

Luddington RJ (2005) Thrombelastography/thromboelastometry. Clin Lab Haematol 27: 81-90.

Nowshad N, Saghafinia M, Panahi F, Bolandparvaz S, Tanideh N (2012) Comparison of the efficacy of two local haemostatic agents. Trauma Mon 16: 188-190.

Paltrinieri S, Meazza C, Giordano A, Tunesi C (2008) Validation of thromboelastometry in horses. Vet Clin Pathol 37: 277-285.

Rall JM, Cox JM, Songer AG, Cestero RF, Ross JD (2013) Comparison of novel hemostatic dressings with QuikClot combat gauze in a standardized swine model of uncontrolled hemorrhage. J Trauma Acute Care Surg 75 (Suppl 2): S150-S156.

Rhee P, Brown C, Martin M, Salim A, Plurad D, Green D, Chambers L, Demetriades D, Velmahos G, Alam $\mathrm{H}$ (2008) QuikClot use in trauma for hemorrhage control: case series of 103 documented uses. J Trauma 64: 1093-1099.

Sobiech P, Radwińska J, Krystkiewicz W, Snarska A, Stopyra A (2008) Changes in the coagulation profile of cattle with left abomasal displacement. Pol J Vet Sci 11: 301-306. 